\title{
Progressive breast fibrosis caused by extreme radiosensitivity: Oncocytogenetic diagnosis and treatment by reconstructive flap surgery
}

\author{
Norbert Mészáros ${ }^{1,2}$ (D) | Gyöngyi Farkas ${ }^{3}$ | Gábor Székely ${ }^{3}$ | Zsuzsa S. Kocsis ${ }^{3}$ | \\ Péter B. Kelemen ${ }^{4}$ | János Fodor ${ }^{1}$ | Csaba Polgár ${ }^{1,2}$ | Zsolt Jurányi ${ }^{3}$
}

\footnotetext{
${ }^{1}$ Centre of Radiotherapy, National Institute of Oncology, Budapest, Hungary

${ }^{2}$ Department of Oncology, Semmelweis University, Faculty of Medicine, Budapest, Hungary

${ }^{3}$ Department of Radiobiology and Diagnostic Onco-Cytogenetics, Centre of Radiotherapy, National Institute of Oncology, Budapest, Hungary

${ }^{4}$ Department of Breast and Sarcoma Surgery, National Institute of Oncology, Budapest, Hungary

Correspondence

Norbert Meszaros, Centre of Radiotherapy, National Institute of Oncology, Rath Gyorgy u. 7-9, Budapest $\mathrm{H}-1122$, Hungary.

Email: meszarosnorbert@oncol.hu
}

\begin{abstract}
Background: Fibrosis, a proliferative response of fibrocytes after tissue injury, is a common sequela of external radiotherapy and can vary greatly among patients even in the absence of DNA repair syndromes, due to their different intrinsic radiosensitivity. Fibrosis is also a serious cosmetic problem for patients, and in some cases it can also imply pain.
\end{abstract}

Case: Here, we report a case of a severe fibrosis 2 years after breast conserving surgery and postoperative 3D conformal breast irradiation. Furthermore, our patient had the suspicion of tumour recurrence. Our examinations were aimed at diagnosing recurrence or the lack of recurrence and investigating whether the symptoms occurred due to overdosing or extreme intrinsic radiosensitivity. Therefore, examining the patients' radiosensitivity, a cytogenetic test was performed, which revealed the patient's increased susceptibility to ionizing radiation, and therefore we rejected the prospect of overdosage. As a solution for the fibrosis, mastectomy was effectuated, and a latissimus dorsi musculocutaneous flap was used for reconstruction.

Conclusions: We suggest a multi-disciplinary approach to manage fibrosis and propose cytogenetic markers to be used as predictors to identify patients who most likely benefit from a certain therapeutic regimen in terms of reduction of therapy-related side effects.

KEYWORDS

breast cancer, chromosome aberrations, cytogenetic analysis, fibrosis, radiotherapy

\section{I INTRODUCTION}

Breast conservation therapy is a preferable option for women with early-stage breast cancer. It consists of resection of the primary breast tumour then followed by whole breast irradiation with a total dose of 45 to $50 \mathrm{~Gy}$. The principal long-term side effect of breast radiotherapy is fibrosis, a serious cosmetic and psychological problem that might be a painful condition for the patient. Besides fibrosis telangiectasis, lymphoedema in the arm and breast oedema occur, and patients often experience moderate or severe pain in the breast, arm, or shoulder. ${ }^{1,2}$
Radiotherapy of breast cancer can also induce late complications in pulmonary and cardiac tissue leading to pulmonary apical fibrosis, ${ }^{3}$ pericardial disease, myocardial fibrosis, coronary artery disease, and valvular lesions. ${ }^{4}$ This aspect of therapeutic intervention is especially accentuated nowadays, when due to the early diagnosis and effective treatments, mortality from breast cancer is decreasing; however, reducing late toxicity is becoming increasingly important.

Fibrosis represents a proliferative response of the surviving fibrocytes due to growth factors released after tissue injury caused by ionizing radiation. Histological and immunological signs of 
radiation-induced fibrosis include the presence of inflammatory infiltrate, macrophages, fibroblasts, matrix proteins, collagen, and growth factor beta 1 (transforming growth factor), ${ }^{1}$ which regulates epidermal growth factor, fibroblast growth factor, tumour necrosis factor, and interleukin-1 production. ${ }^{5}$ While healing of a simple tissue injury might seem a prospective process in time, fractionated radiation therapy produces a series of repeated trauma for tissues. Therefore, during the course of radiation therapy, many steps of the inflammatory process have been repeated leading to a cumulative response. ${ }^{6} \mathrm{~A}$ retrospective study proved that age and the presence of allergy (which includes constant inflammation) were significantly associated with susceptibility to breast fibrosis. ${ }^{7}$

Depending on the late responses of normal tissue, induration, tissue hardening, and changes in shape and volume of the treated breast might appear. Radiation fibrosis and telangiectasia might occur after radiotherapy, but severe cases due to intrinsic sensitivity are rare. In this article, we do not want to compare our case with syndromatic cases such as seen in Ataxia telangiectesia, Fanconi anaemia, Xeroderma pigmentosum patients. In those cases, one already proven gene deficiency causes several symptoms and radiosensitivity also. On the other hand, in cases phrased as intrinsic radiosensitivity cases, there is no sign of radiosensitivity in advance, and it is coded in a complex unknown genetic pattern.

In a multicentre study including 502 breast cancer patients, $86.4 \%$ showed late side effects 3 years after radiotherapy, of which $47.8 \%$ of patients were grade $1,13.4 \%$ grade 2 , and $0.6 \%$ grade $3+$ fibrosis. ${ }^{8}$ Others published $6 \%,{ }^{9} 12.6 \%,{ }^{10}$ and even $23 \%{ }^{11}$ of $\geq$ grade 2 fibrosis. In a study where 47 patients were treated without additional chemotherapeutic drugs after lumpectomy, 30 patients showed grade 1, 12 patients grade 2, and 1 patient grade 3 skin reaction. ${ }^{12}$ In a study of Hoeller et al, 87 breast cancer patients were studied to reveal the relationship of chromosome aberrations and fibrosis. Ten patients developed fibrosis grade 2 or 3 in 1 to 8 years, and patients with more lethal ( $\geq 6.8$ dicentric and acentric fragments) chromosome aberrations showed a higher annual fibrosis rate than patients with low and intermediate chromosome aberration rates. ${ }^{13}$ In general, Azria et al stated that in all radiotherapy patients $5 \%$ to $10 \%$ of the patients develop severe toxicities, decreasing their quality of life. ${ }^{14}$

Differential diagnosis and the management of severe fibrosis is challenging to physicians, radiologists, radiobiologist, and surgeons. We report a case of progressive breast fibrosis in a 61-year-old woman treated by 3D conformal breast irradiation, studied by radiobiological methods and treated by reconstructive surgery. Complex care by cytogenetic investigation combined with reconstructive surgery of radiotherapy-treated patients has not been published in the literature so far.

\section{1 | Case}

A Caucasian female patient (age 61) without any history of cancer was referred to our institution in August 2014 for a non-palpable cancer in her right breast, detected by mammography screening. Core biopsy proved invasive ductal carcinoma. The Multidisciplinary Tumour Board indicated radioguided occult lesion localization excision and sentinel node biopsy (tumour $15 \mathrm{~mm}$, grade III, without LVI, ER 100\%, PR 15\%, Ki67 17\% to 18\% Her2neg., negative sentinel lymph node).

After surgery (September 2014), the patient had no complains, and the cosmetic result was satisfactory. Between November 2014 and January 2015, right sided whole breast irradiation was administered by CT-based, 3D conformal technique with a total dose of 50 Gray (Gy) in 25 daily fractions of 2 Gy, five times a week. The breast PTV volume was $533 \mathrm{~cm}^{3}$, and the maximum dose was $57.44 \mathrm{~Gy}$. As acute toxicity, Grade II erythema occurred that was treated with oily cream. Subcutaneous oedema and pain was not observed. Endocrine therapy (aromatase inhibitor) was introduced after irradiation. After 8 weeks, the patient had no complaints, and acute skin side effects disappeared. After 4 months, right breast skin became hyperpigmented, and mildly dense tissue in the lower quadrants was palpable. Two months later (6 months after radiotherapy), this palpable subcutaneous abnormality became moderate, and breast pain occurred. Staging examinations and tumour markers were negative. Seven months after irradiation, breast MRI was performed due to breast pain: on the right side, the pectoral major muscle was wider, and concentric skin thickening was observed, but there was no residual tumour, or recurrence. Further systematic control examinations were performed; increased lesion was detected, and the patient complained of pain. One and a half year after radiotherapy, our patient had the suspicion of breast tumour recurrence; however, what MRI examination detected was not tumour but a fibrotic residual gland in the breast with moderate involution. Short-TI Inversion Recovery measurement showed high circular skin thickening due to irradiation. Also, the right pectoral muscle was wider than its counterpart, a possible post-therapeutic consequence. There was no evidence of abnormal contrast enhancement or signal intensity, which might have indicated the presence of tumour tissue. Core-biopsy revealed extensive fibrosis and fat necrosis. In the skin excisions of the breast, the dermis were thickened and scarred; there were some irregular collagen fibre multiplications, decrease in the number or lack of the skin appendages, and dilated lymphatic vessels. In the excisions from the side line area, moderate nodal lymphoplasmocyter infiltration could be seen penetrating the collagen fibres. Mucus deposition was not identifiable. The deeper layer of the dermis was oedematously loosened in some places. There were no signs of malignancy. In October 2016, significant lymph oedema and pain appeared in the right breast due to progressive fibrosis. The skin of the breast became purple at the breast periphery region and the breast tissue shrunk to a third. However, irradiationcaused widening of the pectoral muscle, concentric skin thickening, and diffuse fibrosis were also observed (Figure 1).

Such severe radiogenic fibrosis is unusual (but not unique), and since our laboratory performs combined radiosensitivity experiments in vitro and in clinical studies, we decided to investigate this case. We also aimed to verify or exclude a potential local over dosage and reassure the patient that the symptoms were not associated with tumour recurrence. Therefore, we studied the chromosome aberrations in the lymphocytes of irradiated and non-irradiated blood after culture and preparation. Heparinised peripheral blood obtained from the patient was irradiated for cytogenetic analysis of intrinsic radiosensitivity as follows. ${ }^{15}$ Two millilitre blood was placed in water-filled plastic phantom in cryotubes, and radiation field was planned with 
FIGURE 1 Severe fibrosis of the patient 18 months after radiotherapy

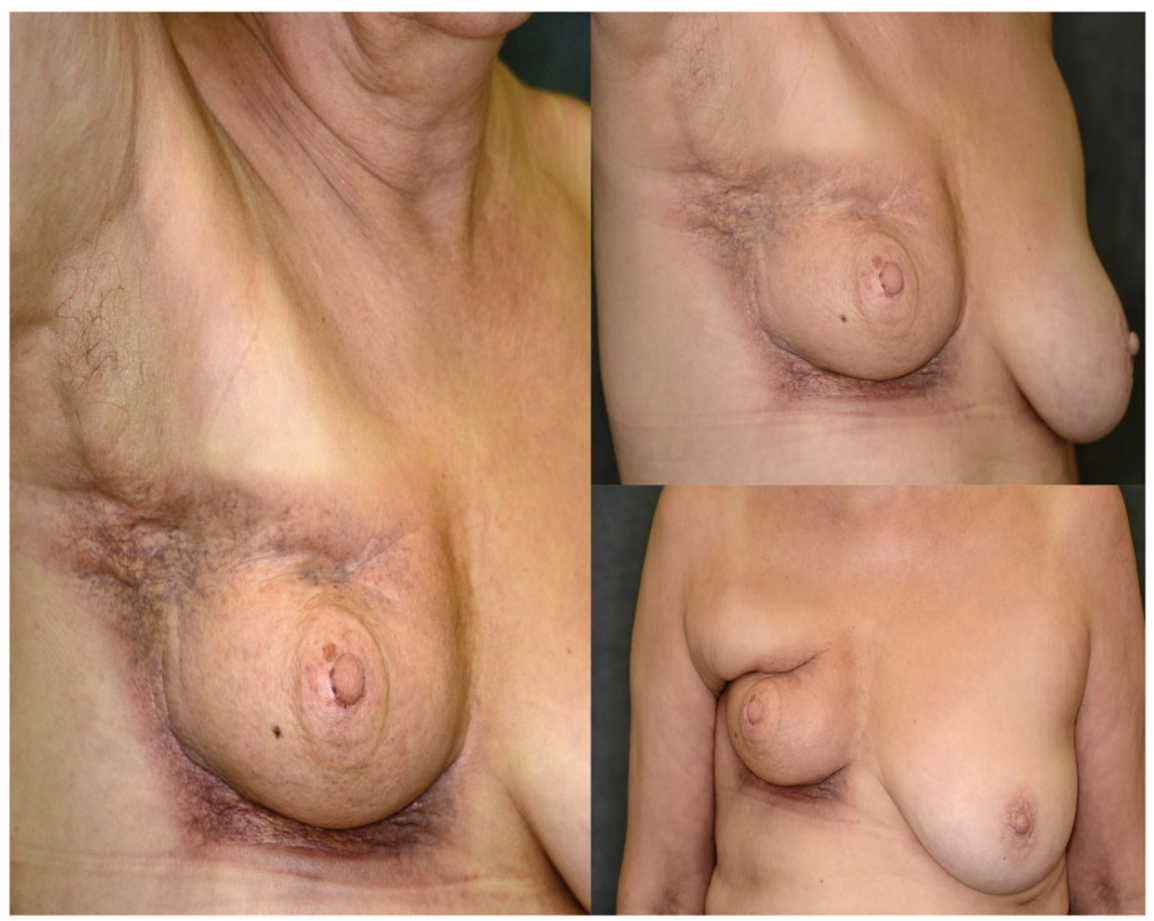

Eclipse TM Treatment Planning System. Irradiation was performed with 3 and 6 Gy at 1400 monitor unit/min (MU/min) and $6 \mathrm{MV}$ on a Varian True-Beam linear accelerator in flattening filter free mode. ${ }^{16}$ Lymphocytes do not divide in blood, they should be stimulated with phytohaemagglutinin and after 48 hours treated with colcemid, a mitotic (spindle formation) inhibitor. Lymphocytes were suspended in a hypotonic solution and then fixed with methanol:acetic acid solution to precipitate both the proteins and DNA in chromosomes. Smears of cells blocked in metaphase were prepared on glass plates followed by Giemsa staining and microscopic analysis. ${ }^{17}$ Two hundred metaphases on eight plates were analysed; in all metaphases, all aberrations were counted, and the aberrations of a hundred metaphases were summed. The standard error of mean was calculated between the groups consisting of a hundred cells. (Figure 2 shows an aberrant cell of the patient and a healthy cell of a control individual.) Values of healthy and cancerous populations whose blood were irradiated under same conditions (non-small cell lung cancer patients T1a-T2a, NOMO) were matched to our data.

Unirradiated (spontaneous) chromosomal aberration values of the patient showed 12-fold (significant) and 2.5-fold (non-significant) increase in the number of dicentric + ring chromosomes, when they were compared with healthy $(N=5)$ and cancerous controls $(N=5)$, respectively (Figure $3 \mathrm{~A})$. The numbers of unirradiated total aberrations were 2.7-fold and 13-fold higher than of healthy and cancerous control groups, respectively (both differences are significant) (Figure 3B). Moreover, the patient's samples irradiated with 3 and 6 Gy in vitro showed more than 1.2-fold total aberrations and more than 1.7-fold dicentric + ring aberrations than any matched controls. The differences are significant in case of the 3-Gy irradiation for total aberrations and in case of 3 and 6 Gy for dicentric + ring chromosomes.

These results suggested that our patient had intrinsic radiosensitivity, which caused severe fibrosis in the breast after standard radiotherapy. Our analysis showed that there was no dosimetric miscalculation, and it was important to verify, because based on these results the patient has been advised, that she should not get any further radiotherapy or radiation-based imaging methods (eg, bone or brain metastases irradiation or CT examinations).

Due to the patient's severe complaints, mastectomy, ablation of the scabby tissue, and replacing the damaged tissue with one of the dorsal lobe were recommended by the Multidisciplinary Tumour Board. Tissues affected by progressive fibrosis were removed, the inner scars were released, scabby parts of the pectoral muscle were
FIGURE 2 Representative pictures of chromosomes of our patient $(A)$ and of a healthy control $(B)$
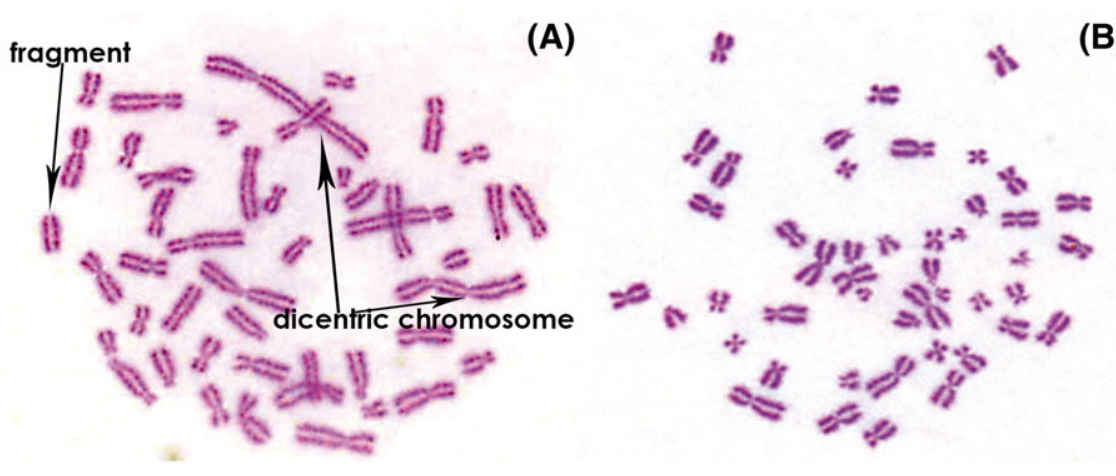

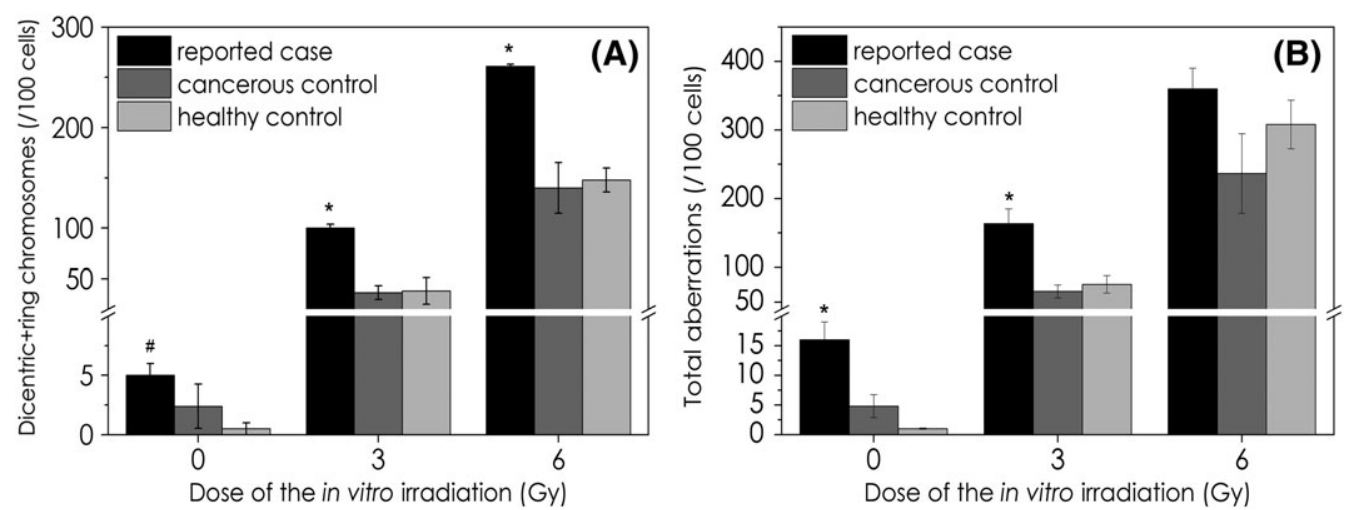

FIGURE 3 Number of dicentric + ring chromosomes (A) and total aberrations (B) in unirradiated blood and after 3 and 6 Gy in vitro irradiation. (Data are marked with * if values of our patient are significantly different from both healthy and cancerous control and marked with \# if they are significantly different from healthy control. ANOVA analysis is made in Statistica 7 software with the parameters: $\mathrm{Cl}=95 \%, P<0.05$ )

removed, and a latissimus dorsi musculocutaneous flap was used for reconstruction. No complications occurred, and the cosmetic result was satisfactory (Figure 4). On control 2 months after the surgery, there were no complaints, and the patient was able to raise her arm above her head, a movement impossible due to fibrosis before surgery.

\section{2 | DISCUSSION}

Radiobiology research focused for decades to find promising biomarkers in severe fibrosis cases which cannot be explained with single gene deficiences (ATM, XPA). Cytogenetic methods provided positive predictive value in numerous, but small cohort studies. For example, micronucleus tests were performed on the blood sample of 38 prostate cancer patients taken before radiotherapy which were irradiated with 1 to $4 \mathrm{~Gy}$. The lymphocytes of the patients who developed more severe radiogenic toxicities after radiotherapy showed significantly more micronucleus number than patients' without severe side effects. This effect was measurable only with more than 2-Gy irradiation of the blood samples. ${ }^{18}$ However, micronucleus method is less sensitive than chromosome aberration technique. ${ }^{19}$ In a previously mentioned study with 47 breast cancer cases using 3-Gy in vitro irradiation before therapy, patients with more translocations (proved by FISH technique) showed less latency period before late skin effects appeared. ${ }^{12}$ In a cohort study including 87 cases of breast cancer patients, it was shown that 6-Gy but not 3-Gy irradiation of patients' blood before radiotherapy induced chromosome aberrations and was

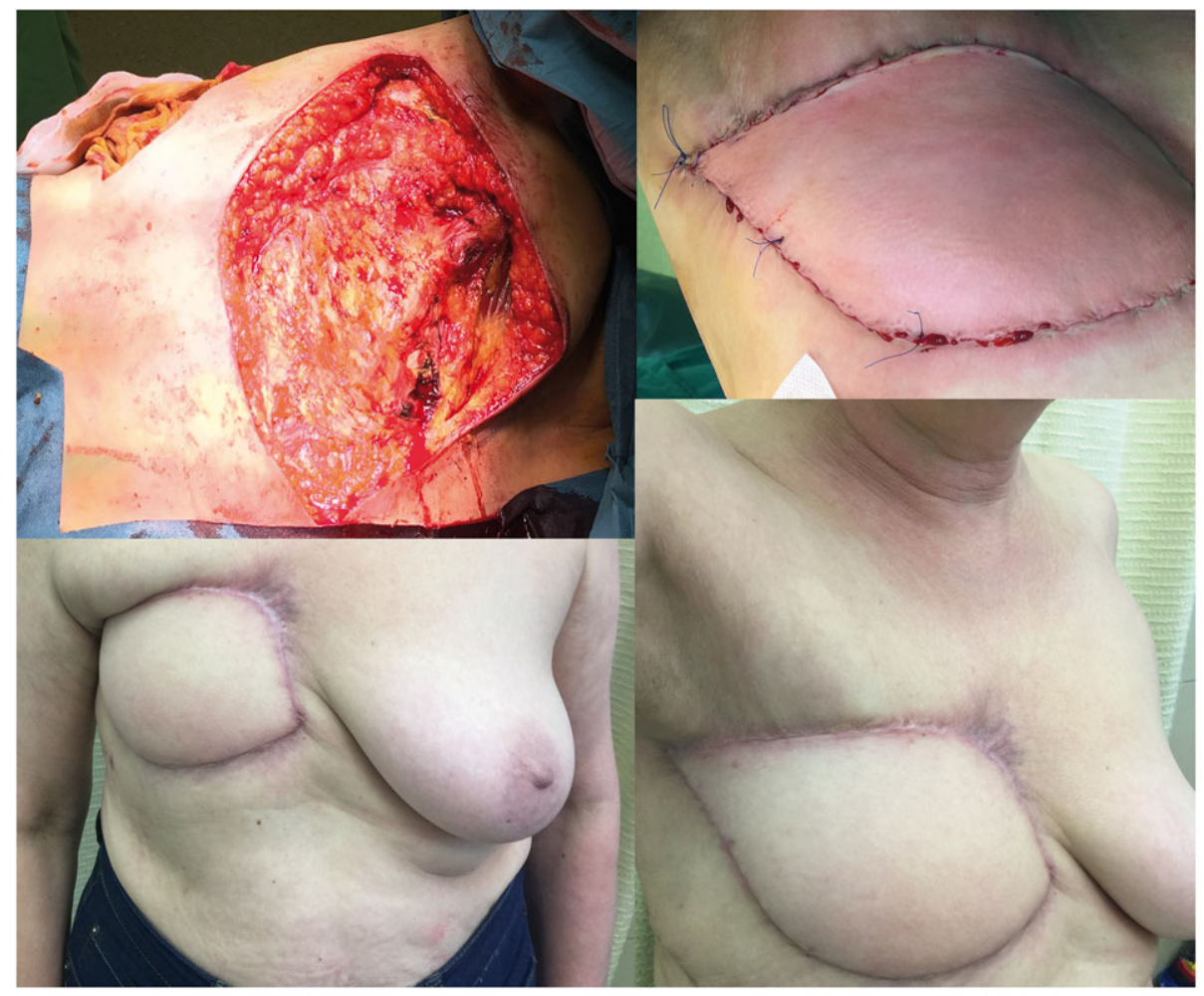

FIGURE 4 Onco-plastic surgery with dorsal lobe reconstruction 
in good correlation with acute side effects of the skin. ${ }^{15}$ In another study, radiation of lymphocytes in G2 phase was not found to be a good predictor of either acute or late toxicities in 72 larynx tumour patients. $^{20}$ The chromosome aberration method is cheap but highly labour intensive, which could limit its wider use. On the other hand, it is expected that in the future automatization will be possible, as there are attempts to achieve this purpose. ${ }^{21,22}$

It is widely accepted that in most cases no single gene is responsible for radiosensitivity; therefore, research is focused on gene expression and single polymorphism patterns, but both are highly expensive. On the other hand, "Radiation-induced CD8 T-lymphocyte Apoptosis" method has negative predictive value and marks patient population with no radiation toxicity risk..$^{8,23,24}$ The method uses lymphocytes as normal tissue surrogate and has been tested in large clinical cohort and at various tumour sites. This method revealed that the risk of fibrosis was decreased in non-smoking population and those without adjuvant hormone therapy. (Our patient was treated with aromatase inhibitor after radiotherapy and was a non-smoker.)

On the basis of literature data and our experience, we propose cytogenetical characterisation of cancer patients before their radiotherapy may identify patients with increased risk of late side effect.

These patients could be treated with mastectomy to avoid irradiation, and oncoplastic reconstruction or with brachytherapy, which has been shown to cause less toxicity, and might get tamoxifen free hormone therapy or newer chemotherapy regimens with taxane. ${ }^{11}$ Cessation of smoking is even more advisable for this group of patients. Recently, new radiation mitigators and normal tissue radio protectors are under investigation. ${ }^{25}$

In the case of potential radiation overexposure accidents, the use of chromosomal aberration assay is also advisable after radiotherapy, for example in the case of severe late toxicities. Radiation-induced fibrosis occurring more than a decade after breast cancer therapy with the suspicion of recurrent breast tumour has been reported in the literature. ${ }^{26}$ Therefore, patients with the suspicion of tumour recurrence that has been precluded by biopsy may also benefit because further ionising load (X-ray, CT scans) can be reduced.

\section{3 | CONCLUSIONS}

Our research has shown that chromosomal aberration technique can help getting information about the cause of radiogenic progressive fibrosis and plays a role in the decision of further radiotherapy or the choice of imaging methods. We propose the use of this methodology for the investigation of other radiation toxicities and even before radiotherapy to detect patients more susceptible for late toxicities. The most important message of our work is that a multidisciplinary approach can manage even a complicated case. It is also demonstrated that reconstructive surgery can solve severe fibrosis cases for the satisfaction of the patient.

\section{4 | ETHICAL STATEMENT}

The study was approved by the Local Ethics Committee in strict compliance with the Declaration of Helsinki. Patient's written consent was obtained for publication of this case report.

\section{AUTHORS' CONTRIBUTIONS}

All authors had full access to the data in the study and took responsibility for the integrity of data and accuracy of the data analysis. Conceptualization, N.M. and J.F.; Methodology, G.F; G.S., and P.B.K.; Investigation, Z.S.K.; Writing-Original Draft, N.M.; Writing-Review \& Editing, Z.J.; Supervision, C.P.

\section{ACKNOWLEDGEMENT}

We highly acknowledge the contribution of Dr Zsuzsa Sándor, who analysed the pathologic samples of our patient. Furthermore, special thanks for Krisztina Kiss technician for the preparation of the chromosome samples.

\section{CONFLICT OF INTEREST}

Norbert Meszaros, Gyongyi Farkas, Gabor Szekely, Zsuzsa S. Kocsis, Peter Bertalan Kelemen, Janos Fodor, Csaba Polgar, and Zsolt Juranyi state that there are no conflicts of interest.

\section{ORCID}

Norbert Mészáros (10 http://orcid.org/0000-0002-1570-7565

\section{REFERENCES}

1. Hille-Betz U, Vaske B, Bremer M, et al. Late radiation side effects, cosmetic outcomes and pain in breast cancer patients after breastconserving surgery and three-dimensional conformal radiotherapy: risk-modifying factors. Strahlenther Onkol. 2016;192(1):8-16.

2. Goffman TE, Laronga C, Wilson L, Elkins D. Lymphedema of the arm and breast in irradiated breast cancer patients: risks in an era of dramatically changing axillary surgery. Breast J. 2004;10(5):405-411.

3. Seam RK, Revannasiddaiah S, Bhardwaj B, Gupta MK. Pulmonary apical fibrosis in a patient treated earlier for breast cancer. BMJ Case Rep. $2013 ; 2013$.

4. Liu LK, Ouyang W, Zhao X, et al. Pathogenesis and prevention of radiation-induced myocardial fibrosis. Asian Pac J Cancer Prev. 2017; 18(3):583-587.

5. Hojan K, Milecki P. Opportunities for rehabilitation of patients with radiation fibrosis syndrome. Rep Pract Oncol Radiother. 2014;19(1):1-6.

6. Denham JW, Hauer-Jensen M. The radiotherapeutic injury-a complex 'wound'. Radiother Oncol. 2002;63(2):129-145.

7. Lilla C, Ambrosone CB, Kropp S, et al. Predictive factors for late normal tissue complications following radiotherapy for breast cancer. Breast Cancer Res Treat. 2007;106(1):143-150.

8. Azria D, Riou O, Castan F, et al. Radiation-induced CD8 T-lymphocyte apoptosis as a predictor of breast fibrosis after radiotherapy: results of the prospective multicenter French trial. EBioMedicine. 2015;2(12): 1965-1973.

9. Bourgier C, Castan F, Riou O, et al. Impact of adjuvant hormonotherapy on radiation-induced breast fibrosis according to the individual radiosensitivity: results of a multicenter prospective French trial. Oncotarget. 2018;9(21):15757-15765.

10. Collette S, Collette L, Budiharto T, et al. Predictors of the risk of fibrosis at 10 years after breast conserving therapy for early breast cancer: a study based on the EORTC trial 22881-10882 'boost versus no boost'. Eur J Cancer. 2008;44(17):2587-2599.

11. Johansen J, Overgaard J, Overgaard M. Effect of adjuvant systemic treatment on cosmetic outcome and late normal-tissue reactions after breast conservation. Acta Oncol. 2007;46(4):525-533. 
12. Huber R, Braselmann H, Geinitz H, et al. Chromosomal radiosensitivity and acute radiation side effects after radiotherapy in tumour patientsa follow-up study. Radiat Oncol. 2011;6(1):32.

13. Hoeller $\mathrm{U}$, Borgmann $\mathrm{K}$, Bonacker $\mathrm{M}$, et al. Individual radiosensitivity measured with lymphocytes may be used to predict the risk of fibrosis after radiotherapy for breast cancer. Radiother Oncol. 2003;69(2): 137-144.

14. Azria D, Lapierre A, Gourgou S, et al. Data-based radiation oncology: design of clinical trials in the toxicity biomarkers era. Front Oncol. 2017;7:83.

15. Borgmann K, Hoeller U, Nowack S, et al. Individual radiosensitivity measured with lymphocytes may predict the risk of acute reaction after radiotherapy. Int J Radiat Oncol Biol Phys. 2008;71(1):256-264.

16. Vassiliev ON, Titt U, Ponisch F, et al. Dosimetric properties of photon beams from a flattening filter free clinical accelerator. Phys Med Biol. 2006;51(7):1907-1917.

17. Gundy S, Varga LP. Chromosomal aberrations in healthy persons. Mutat Res. 1983;120(2-3):187-191.

18. Lee TK, Allison RR, O'Brien KF, et al. Lymphocyte radiosensitivity correlated with pelvic radiotherapy morbidity. Int J Radiat Oncol Biol Phys. 2003;57(1):222-229.

19. Perumal V, Gnana Sekaran TS, Raavi V, et al. Radiation signature on exposed cells: relevance in dose estimation. World J Radiol. 2015;7(9):266-278.

20. Lisowska H, Lankoff A, Wieczorek A, et al. Enhanced chromosomal radiosensitivity in peripheral blood lymphocytes of larynx cancer patients. Int J Radiat Oncol Biol Phys. 2006;66(4):1245-1252.
21. Finnon P, Lloyd D. A preliminary evaluation of the Edinburgh dicentric hunter. J Radiat Res. 1992;33(Suppl):215-221.

22. Bayley R, Carothers A, Chen X, et al. Radiation dosimetry by automatic image analysis of dicentric chromosomes. Mutat Res. 1991;253(3): 223-235.

23. Bordon E, Henriquez-Hernandez LA, Lara PC, et al. Role of CD4 and CD8 T-lymphocytes, B-lymphocytes and natural killer cells in the prediction of radiation-induced late toxicity in cervical cancer patients. Int J Radiat Biol. 2011;87(4):424-431.

24. Foro $\mathrm{P}$, Algara M, Lozano J, et al. Relationship between radiationinduced apoptosis of $\mathrm{T}$ lymphocytes and chronic toxicity in patients with prostate cancer treated by radiation therapy: a prospective study. Int J Radiat Oncol Biol Phys. 2014;88(5):1057-1063.

25. Maier $P$, Wenz F, Herskind $C$. Radioprotection of normal tissue cells. Strahlenther Onkol. 2014;190(8):745-752.

26. Sarsenov D, Aktepe F, Ozmen V. Radiation fibrosis syndrome imitating breast cancer recurrence; a case report. J Breast Health. 2017;13(1):40-42.

How to cite this article: Mészáros N, Farkas G, Székely G, et al. Progressive breast fibrosis caused by extreme radiosensitivity: Oncocytogenetic diagnosis and treatment by reconstructive flap surgery. Cancer Reports. 2019;2:e1126. https:// doi.org/10.1002/cnr2.1126 